\title{
Development of Standard Operating Protocol for Slide Preparation of Powdered Bark Samples with Varying Grinding Techniques
}

\author{
Dharya Singh, Vidhu Aeri*, DB Ananthanarayana
}

Dharya Singh, Vidhu Aeri*, DB Ananthanarayana

Department of Pharmacognosy and Phytochemistry, Faculty of Pharmacy, Jamia Hamdard, New Delhi-62, INDIA.

\section{Correspondence}

\section{Vidhu Aeri}

Department of Pharmacognosy and Phytochemistry, Faculty of Pharmacy, Jamia Hamdard, New Delhi-62, INDIA.

Phone No: +91-9873496942

E-mail: vidhuaeri@yahoo.com

\section{History}

- Submission Date: 29-08-2017;

- Review completed: 20-11-2017;

- Accepted Date: 19-12-2017

DOI : 10.5530/pj.2018.2.47

\section{Article Available online}

http://www.phcogj.com/v10/i2

\section{Copyright}

(C) 2018 Phcog.Net. This is an openaccess article distributed under the terms of the Creative Commons Attribution 4.0 International license.

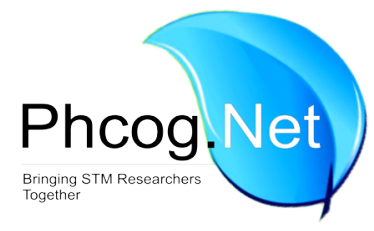

\begin{abstract}
Background: Powder microscopy is the most common method to authenticate herbal drugs. The botanical origin and quality can be determined based on cellular features. Objective: To develop a standard operating protocol to prepare slides of powdered bark samples obtained by using different grinding techniques. Materials and methods: Alstonia scholaris, Saraca asoca, Syzygium cumini, Terminalia arjuna and Pongamia pinnata were selected for study. Three samples of each bark were studied: Sample A was collected from NISCAIR Pusa, New Delhi. Sample B and C were procured from industries and were coded as: AP I, AP II, AP III, AP IV and $A P V$ and MP I, MP II, MP III, MP IV and MP V respectively. The slides were prepared by soaking and chemical treatment and photographed by Motic microscope. The samples were observed for general and specific features of the bark and the impact of grinding (industrial grinding and blade grinding in laboratory) on probability of change in various microscopical features. Results: The microscopical features were found to be stable and specific, which can be used to determine the botanical identity of the drug. There was no significant difference in microscopical characters of powdered barks which emphasized that grinding technique does not have any impact on powder microscopy of different bark samples. The method of preparation of slide was also optimized. Conclusion: Powder microscopy, irrespective of grinding technique can be used as a cheap, fast, efficient and accurate method to authenticate herbs or distinguishing species with similar morphological characters without cutting and examining sections.

Key words: Powder microscopy, Grinding technique, Slide optimization.
\end{abstract}

\section{INTRODUCTION}

The increase in demand of herbal medicines worldwide underlines the importance of fast, accurate and efficient method to authenticate a crude drug. The increased market has generated an increase in counterfeit drugs. The unique problems of authentication are related to species confusion of herbs and one name being shared by many herbs and one herb having different names. Poisoning incidents have been reported due to confusion and have raised international concern. Therefore, correct botanical identity is of utmost priority to the safe and effective use of herbs.

Today, a variety of methods are available to authenticate crude drugs, ranging from simple morphological examination to physical and chemical analysis, and DNA molecular biology. Each method has its limitations. However, ordinary light microscopy is still the most practical method for primary authentication. ${ }^{1}$ It has been commonly used to authenticate herbal medicines in India and many other countries because of its advantage of small amount of sample required, fast speed and low cost. Furthermore, herbal medicines are mostly low-cost medicine, should not be raised in price because of usage of unnecessary highly sophisticated methods for authentication. The complex chemical nature of herbs and their products require adequate quality control for herbal drug manufacturers and food and beverages industries and personal care also. In addition, quality of botanical products and adulteration in the supply chain make it necessary to address the identity issues in a comprehensive manner. The natural products industry is growing in both complexity and compliance. The compliance requires more testing and the best way to work with a lab to minimize complications are crucial to understand.

Adulteration and substitution of herbal drugs can cause serious health problems to consumers. The first step for quality control and authentication of any herbal drug is to study its morphology followed by its anatomy or microscopy. Powdering of crude drugs in industries is a dust generating process. Ayurvedic herbal industries are also shifting towards GMP. Therefore, industries are willing to purchase grounded herbs, which make it necessary to check its botanical identity at first stage. Many reference books record the powder microscopic characters of 
herbal drugs. For example, Chinese Materia Medica $^{2}$ European Pharmacopoeia, ${ }^{3}$ British Pharmacopoeia, ${ }^{4}$ United States Pharmacopeia, ${ }^{5}$ Japanese Pharmacopoeia, ${ }^{6}$ Ayurvedic Pharmacopoeia of India ${ }^{7}$ and Vietnamese Pharmacopoeia. ${ }^{8}$

Industry emphasizes on powder microscopy as these tests are prescribed in pharmacopoeial monographs. Moreover, there is no need to cut sections and minimum expertise and equipment is required. The botanical identity of the plant can be confirmed within seconds. ${ }^{9,10,11}$ Although powder microscopy of herbal drugs has been mentioned in various pharmacopoeias, but description of characters is not exhaustive in nature and very little information is provided about microscopical characteristics of powdered herbal drugs. Alstonia scholaris, Saraca asoca, Terminalia arjuna, Pongamia pinnata and Syzygium cumini have been used in Ayurvedic formulations for a very long time and have proven benefits for treatment of variety of ailments. The present study is focused at development of standard operating procedure for preparation of slides of powdered bark samples with varying grinding techniques - industrial and laboratory blade grinding and comparison of various microscopical features like sclereids and stone cells, calcium oxalate crystals, medullary rays, cork cells, fibres, starch grains etc using Motic microscope among various samples of these commonly used herbal drugs.

\section{MATERIALS AND METHODS}

\section{Collection of samples}

Sample A (100 g) was obtained by pulverizing barks of Alstonia scholaris, Saraca asoca, Syzygium cumini, Terminalia arjuna and Pongamia pinnata, collected from NISCAIR Pusa campus and authenticated by Dr Sunita Garg, Scientist G and coded as NA I, NA II, NA III, NA IV and NA V respectively. Sample B and C (100 g), powdered barks procured from industry and coded as AP I, AP II, AP III, AP IV, AP V and MP I, MP II, MP III, MP IV and MP V respectively.

\section{Chemicals and Reagents}

All the chemicals used in the experiments were of analytical grade. Potassium chlorate was procured from Sigma Aldrich. Potassium chlorate and 50\% nitric acid known as Schulze's Maceration Fluid, ${ }^{3,12}$ a powerful oxidizing agent, removes lignin from vegetable tissues was used to disintegrate hard woody substances such as sclereids, stone cells and fibres.

\section{Organoleptic evaluation}

Organoleptic evaluation of the powder was carried out by examining its colour, odour, state and appearance Table 1.

\section{Method of Preparation of Slides}

Two types of slides were prepared for the visualization of microscopical features present in a bark. The slide preparation method was optimized to determine the dilution of powdered bark in water to visualize common as well as distinguishing characters of the bark.

Slide I: A $500 \mathrm{mg}$ of moderately fine (44/85) and fine (85) powdered material was soaked overnight in $10 \mathrm{ml}$ of water (1:20) for $24 \mathrm{~h}$. Subsequently, the contents were poured in a petriplate and slide was prepared by mounting the contents on a clean and dried slide with a brush and observed under Motic microscope moticam 3.0 MP, AE 2000. Most of the features were visible except stone cells and sclereids, which require treatment by oxidizing agent.

Slide II: The slide was prepared by potassium chlorate treatment which is used as an oxidizing agent used for disruption of stone cells and sclereids. However, calcium oxalate crystals and starch grains are destroyed using this method. A $200 \mathrm{mg}$ of powdered material was boiled with $5 \mathrm{ml}$ of $50 \%$ nitric acid. To this, added a pinch $\sim 100-150 \mathrm{mg}$ of potassium chlorate. Poured the contents in a Petri plate after effervescence ceases. The contents were mounted on a clean and dried glass slide with the help of a brush. Observed under Motic microscope.

\section{RESULTS}

The present study was focused on to study the difference between powder microscopy of moderately fine (44/85) and fine (85) powder procured from industry Table 2 . The powder microscopy of all the samples were same. Although there was a slight difference in few characters. Calcium oxalate crystals were abundantly present in moderately fine powder (laboratory grinding) whereas stone cells and sclereids which are main distinguishing feature for any bark was found to be more clear and prominent in fine powder (industrial grinding) because laboratory grinding was coarser as compared to industrial grounding. None of the

Table 1: Organoleptic properties of various samples.

\begin{tabular}{ccccc}
\hline Sample & Appearance & Colour & Odour & State \\
\hline NA, I & Free flowing & Pale buff brown & Characteristic & Powder \\
NA II & Gritty & Dark brown & Characteristic & Powder \\
NA III & Fibrous & Dark greyish brown & No odour & Powder \\
NA IV & Gritty & Buff & Faint characteristic & Powder \\
NA V & Very fine & Pinkish & Characteristic & Powder \\
AP, I & Free flowing & Buff brown & Characteristic & Powder \\
AP II & Gritty & Pale brown & Characteristic & Powder \\
AP III & Fibrous & Dark brown & No odour & Powder \\
AP IV & Gritty & Buff & Faint characteristic & Powder \\
AP V & Very fine & Pinkish & Characteristic & Powder \\
MP, I & Free flowing & Pale buff & Characteristic & Powder \\
MP II & Gritty & Pale brown & Characteristic & Powder \\
MP III & Fibrous & Dark brown & No odour & Powder \\
MP IV & Gritty & Buff & Faint characteristic & Powder \\
MP V & Very fine & Pinkish & Characteristic & Powder \\
\hline
\end{tabular}


Table 2: Microscopic characters of various samples.

\begin{tabular}{|c|c|c|c|c|c|c|c|c|c|c|c|c|c|c|c|}
\hline $\begin{array}{l}\text { Microscopic } \\
\text { characters }\end{array}$ & NA, I & $\frac{\mathrm{AP}}{\mathrm{I}}$ & MP, I & NA II & AP II & MP II & NA III & AP III & MP III & NA IV & AP IV & MP IV & NA V & APV & MP V \\
\hline \multicolumn{16}{|l|}{ STONE CELLS ${ }^{*}$} \\
\hline N/A & - & - & - & - & - & - & - & - & - & + & + & + & - & - & - \\
\hline Abundant & + & + & + & + & + & + & - & - & - & - & - & - & + & + & + \\
\hline Few & - & - & - & - & - & - & + & + & + & - & - & - & - & - & - \\
\hline Isolated & + & + & + & + & + & + & + & + & + & - & - & - & - & - & - \\
\hline Group & + & + & + & + & + & + & + & + & + & - & - & - & + & + & + \\
\hline Lignified & - & - & - & + & + & + & - & - & - & - & - & - & + & + & + \\
\hline Squarish & - & - & - & + & + & + & + & + & + & - & - & - & + & + & + \\
\hline Oblong & + & + & + & - & - & - & - & - & - & - & - & - & + & + & + \\
\hline Oval & + & + & + & + & + & + & + & + & + & - & - & - & + & + & + \\
\hline Spherical & + & + & + & - & - & - & - & - & - & - & - & - & + & + & + \\
\hline Rectangular & - & - & - & + & + & + & - & - & - & - & - & - & + & + & + \\
\hline Wide lumen & + & + & + & + & + & + & + & + & + & - & - & - & + & + & + \\
\hline Narrow lumen & + & + & + & + & + & + & + & + & + & - & - & - & - & - & - \\
\hline Thick walled & + & + & + & - & - & - & + & + & + & - & - & - & + & + & + \\
\hline Branching pits & - & - & - & - & - & - & - & - & - & - & - & - & + & + & + \\
\hline Radiating pits & + & + & + & + & + & + & + & + & + & - & - & - & + & + & + \\
\hline Striated walls & + & + & + & + & + & + & + & + & + & - & - & - & + & + & + \\
\hline \multicolumn{16}{|l|}{ SCLEREID* } \\
\hline $\mathrm{N} / \mathrm{A}$ & - & - & - & - & - & - & - & - & - & + & + & + & + & + & + \\
\hline Abundant & + & + & + & + & + & + & + & + & + & - & - & - & - & - & - \\
\hline Few & - & - & - & - & - & - & - & - & - & - & - & - & - & - & - \\
\hline Isolated & + & + & + & + & + & + & + & + & + & - & - & - & - & - & - \\
\hline Group & + & + & + & + & + & + & - & - & - & - & - & - & - & - & - \\
\hline Fibrous & - & - & - & + & + & + & + & + & + & - & - & - & - & - & - \\
\hline Thick walled & + & + & + & + & + & + & + & + & + & - & - & - & - & - & - \\
\hline Distinct pits & - & - & - & + & + & + & + & + & + & - & - & - & - & - & - \\
\hline Striations & + & + & + & + & + & + & + & + & + & - & - & - & - & - & - \\
\hline Branched & - & - & - & - & - & - & + & + & + & - & - & - & - & - & - \\
\hline Narrow lumen & + & + & + & + & + & + & + & + & + & - & - & - & - & - & - \\
\hline Wide lumen & + & + & + & + & + & + & + & + & + & - & - & - & - & - & - \\
\hline \multicolumn{16}{|l|}{$\begin{array}{l}\text { MEDULLARY } \\
\text { RAYS }\end{array}$} \\
\hline Tangentially cut & + & + & + & + & + & + & + & + & + & + & + & + & + & + & + \\
\hline Radially cut & - & - & - & + & + & + & + & + & + & + & + & + & + & + & + \\
\hline Longitudinally cut & + & + & + & + & + & + & + & + & + & + & + & + & + & + & + \\
\hline $\begin{array}{c}\text { Rectangular } \\
\text { CORK }\end{array}$ & - & - & - & + & + & + & + & + & + & + & + & + & - & - & - \\
\hline Thick walled & + & + & + & + & + & + & + & + & + & + & + & + & + & + & + \\
\hline Yellowish brown & - & - & - & + & + & + & - & - & - & - & - & - & - & - & - \\
\hline Polygonal & - & - & - & + & + & + & - & - & - & + & + & + & + & + & + \\
\hline Hexagonal & + & + & + & - & - & - & - & - & - & - & - & - & + & + & + \\
\hline Rectangular & + & + & + & + & + & + & - & - & - & - & - & - & - & - & - \\
\hline Squarish & + & + & + & - & - & - & - & - & - & - & - & - & - & - & - \\
\hline Pitted walls & + & + & + & - & - & - & - & - & - & - & - & - & - & - & - \\
\hline Pentagonal & + & + & + & - & - & - & - & - & - & - & - & - & - & - & - \\
\hline Lignified & + & + & + & - & - & - & + & + & + & - & - & - & - & - & - \\
\hline \multicolumn{16}{|l|}{ STARCH GRAINS } \\
\hline Simple & + & + & + & + & + & + & + & + & + & + & + & + & + & + & + \\
\hline Compound & - & - & - & - & - & - & + & + & + & + & + & + & + & + & + \\
\hline
\end{tabular}




\begin{tabular}{|c|c|c|c|c|c|c|c|c|c|c|c|c|c|c|c|}
\hline $\begin{array}{l}\text { Microscopic } \\
\text { characters }\end{array}$ & NA, I & $\frac{A P}{1}$ & MP, I & NA II & AP II & MP II & NA III & AP III & MP III & NAIV & AP IV & MP IV & NAV & AP V & MP V \\
\hline Spherical & + & + & + & + & + & + & - & - & - & - & - & - & + & + & + \\
\hline Triangular & - & - & - & - & - & - & + & + & + & - & - & - & - & - & - \\
\hline Eccentric hilum & - & - & - & - & - & - & + & + & + & - & - & - & - & - & - \\
\hline \multicolumn{16}{|l|}{$\begin{array}{l}\text { CALCIUM } \\
\text { OXALATE } \\
\text { CRYSTALS }\end{array}$} \\
\hline N/A & - & - & - & - & - & - & - & - & - & - & - & - & - & - & - \\
\hline Prismatic & + & + & + & + & + & + & - & - & - & - & - & - & + & + & + \\
\hline $\begin{array}{c}\text { BEADED } \\
\text { PHLOEM } \\
\text { PARENCHYMA }\end{array}$ & + & + & + & - & - & - & - & - & - & - & - & - & - & - & - \\
\hline CRYSTAL FIBRES & - & - & - & + & + & + & - & - & - & - & - & - & - & - & - \\
\hline
\end{tabular}

* shows distinguishing characters specific to a bark, + present, -absent

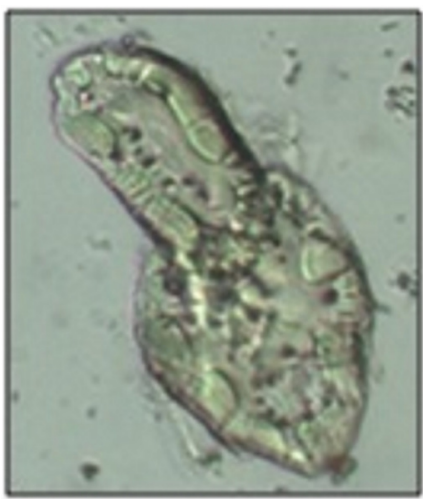

Figure 1: Represents sclereids from NAI.

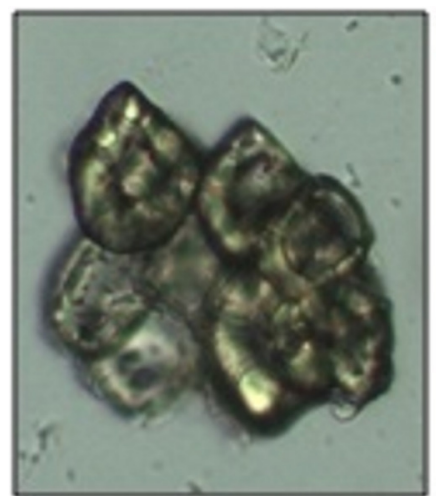

Figure 2: Represents stone cells from NA I.

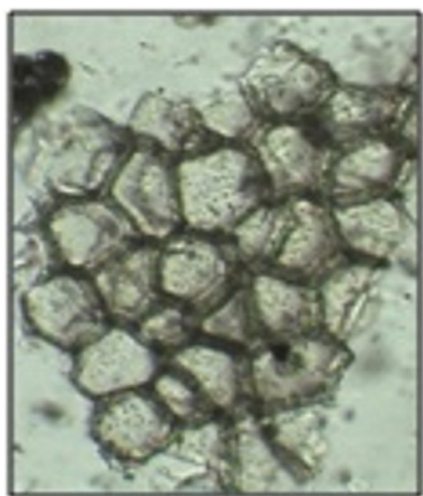

Figure 3: Represents cork with pitted walls from NA I.

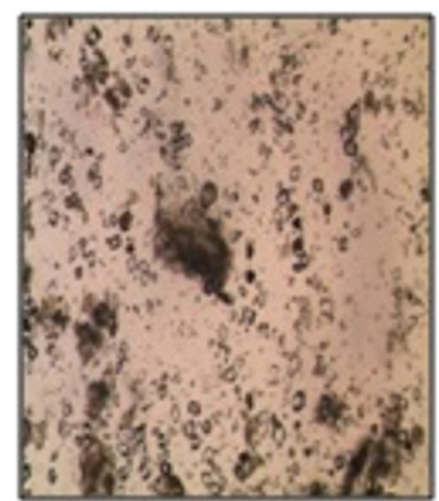

Figure 4: Represents prismatic crystals from NA I. powder characters got destroyed in both moderately fine and fine powder which indicates that grinding technique has no effect on the powder microscopy of the crude drugs. Powdered barks which have high tannin content like Saraca asoca and Syzygium cumini required oxidative treatment by potassium chlorate for visualization of stone cells and sclereids.

Alstonia scholaris is a pale buff brown colour powder having low tannin content. Therefore, overnight soaking of powdered bark was enough for visualization of all features including stone cells and sclereids. As sample received from industry was fine powder. Features were very clear and prominent as compared to moderately fine powder Figure 1- 8 .

Figure 1,2, 3 and 4 represents sclereids, stone cells, cork with pitted walls and prismatic crystals respectively from NA I. Figure 5 and 6 represents prismatic crystals and medullary rays respectively from MP I. Figure 7 and 8 isolated sclereid and group of stone cells respectively from AP I.

Saraca asoca is a pale brown colour powder having high tannin content. Overnight soaking of bark powder was not enough for visualization of features. Therefore, it was treated with potassium chlorate to observe stone cells and sclereids Figure 9-16.

Figure 9, 10, 11 and 12 represents cork cells, group of stone cells, prismatic crystals and fibres from NA II. Figure 13, 14 and 15 represents medullary rays, isolated sclereid and starch grains embedded in a parenchymatous cell from AP II. Figure 16 represents an isolated sclereid from MP II.

Syzygium cumini is a pale grayish brown, fibrous powder. Features were more clear and prominent in fine powder Figure 17-23.

Figure 17 represents rosette crystals from NA III. Figure 18,19, 20, 21 and 22 represents medullary rays, rosette crystals, sclereid, starch grains (blue black due to iodine treatment) and group of stone cells respectively from AP III. Figure 23 represents group of stone cells from MP III.

Terminalia arjuna is a pale pinkish powder. Moderately fine sample was found to be rich source of rosette crystals as compared to fine powder Figure 24-28. 


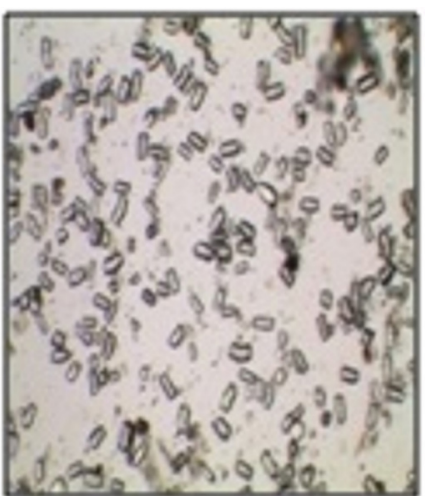

Figure 5: Represents prismatic crystals from MP I.

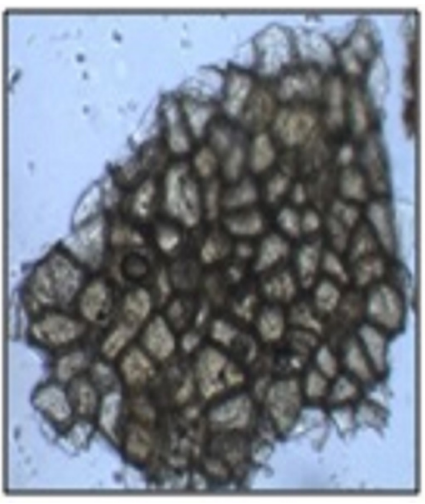

Figure 9: Cork cells from NA II.

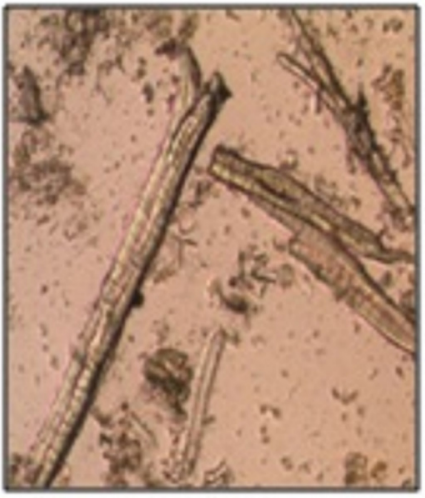

Figure 13: Represents medullary rays from AP II.

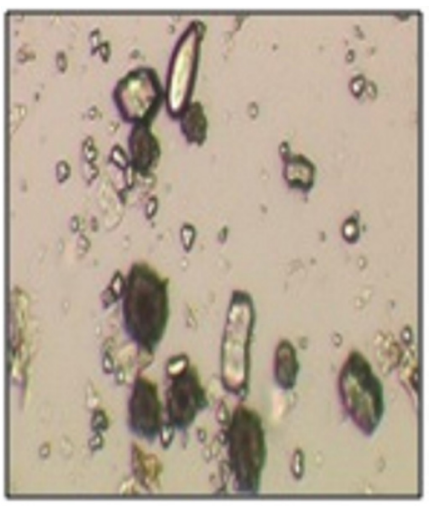

Figure 17: Represents rosette crystals from NA III.

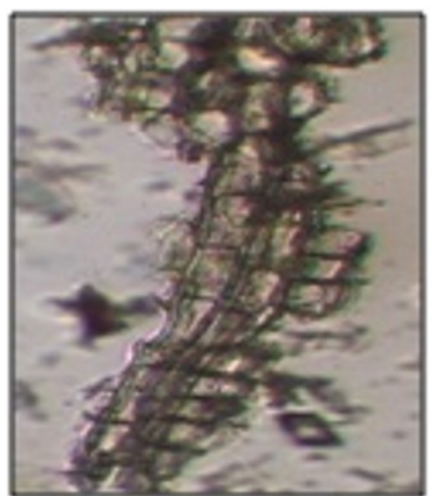

Figure 6: Represents medullary rays from MPI.

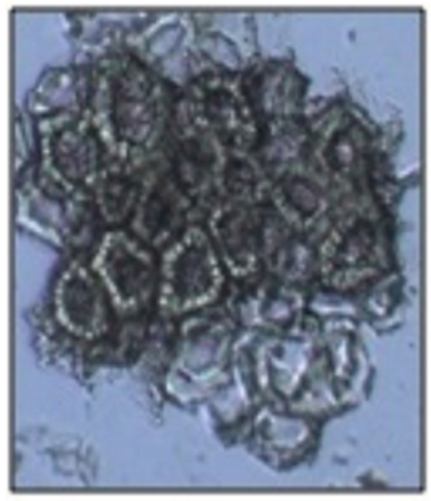

Figure 10: Group of stone cells from NA II.

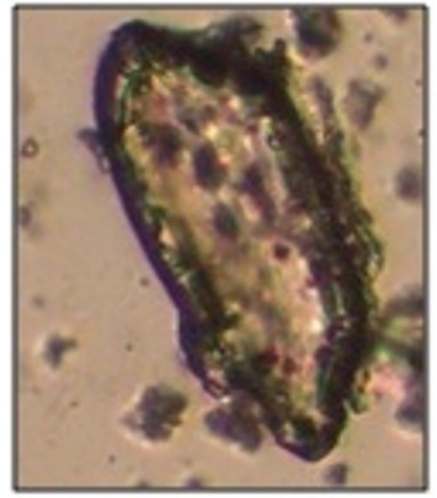

Figure 14: Represents isolated sclereid from AP II.

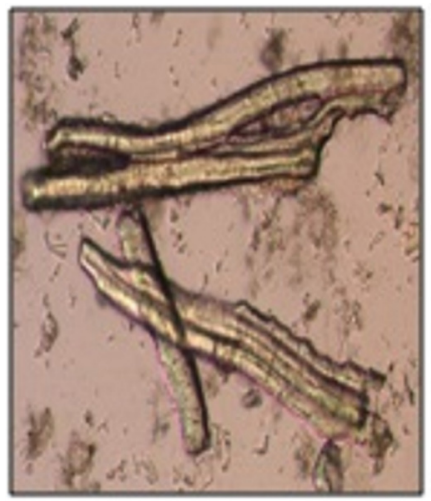

Figure 18: Represents medullary rays from AP III.

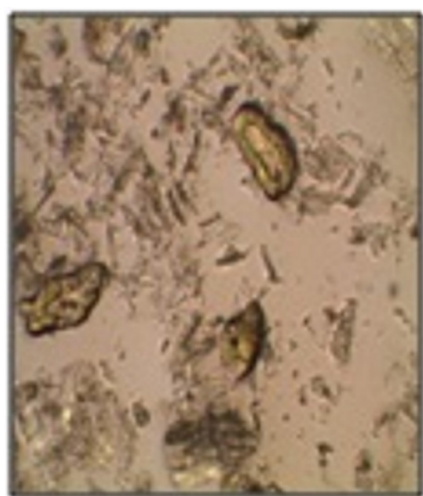

Figure 7: Isolated sclereid from AP I.

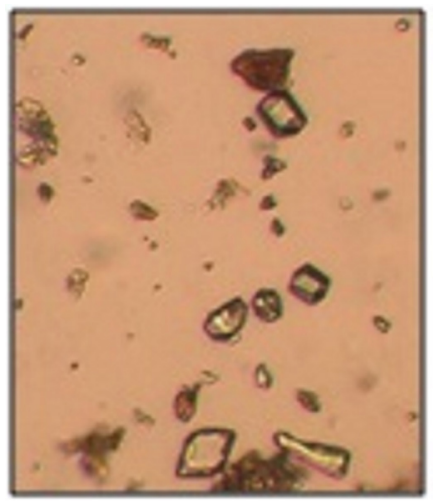

Figure 11: Prismatic crystals from NA II.

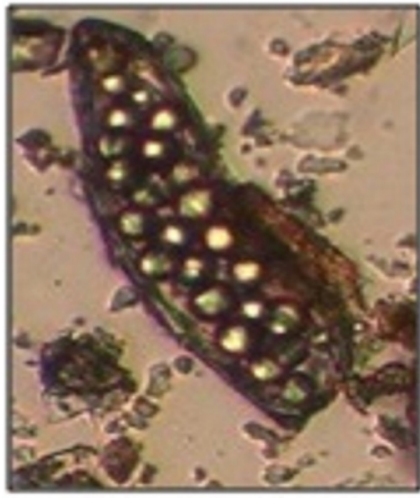

Figure 15: Represents starch grains embedded in a parenchymatous cell from AP II.

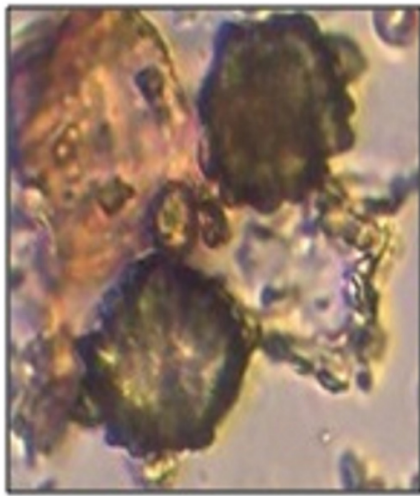

Figure 19: Rosette crystals from AP III.

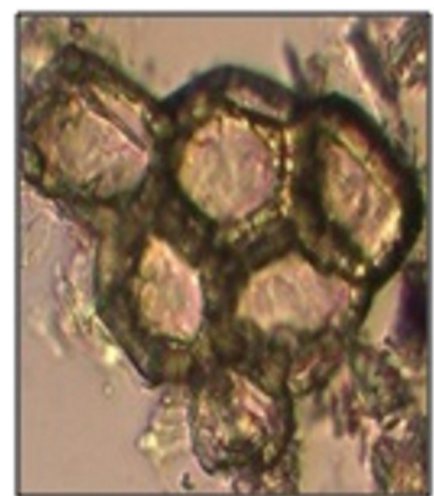

Figure 8: Group of stone cells from API.

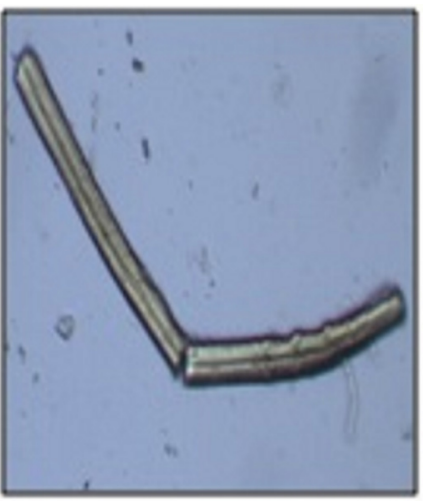

Figure 12: Represents fibre from NA II.

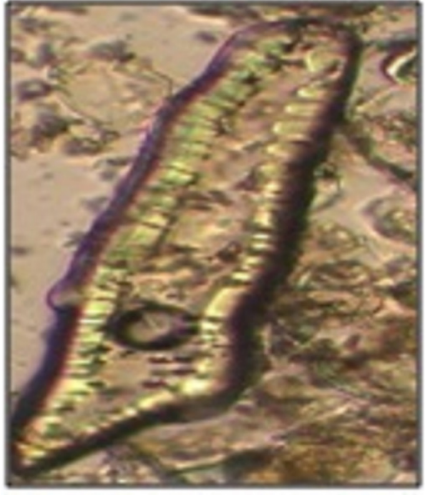

Figure 16: Represents an isolated sclereid from MP II.

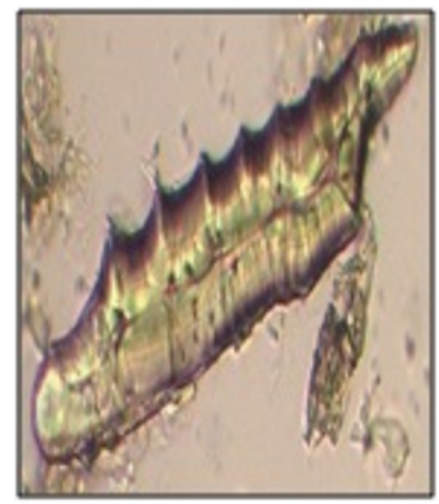

Figure 20: Sclereid from AP III. 


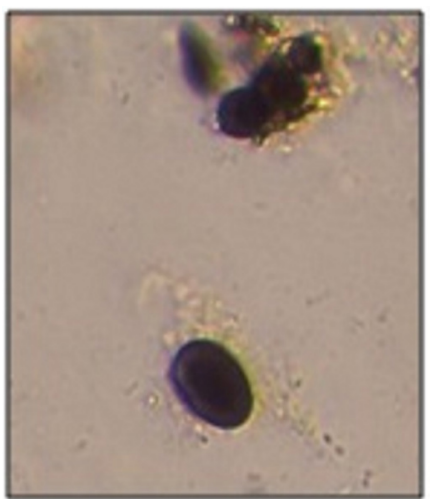

Figure 21: Starch grains (blue black due to iodine treatment) from AP III.

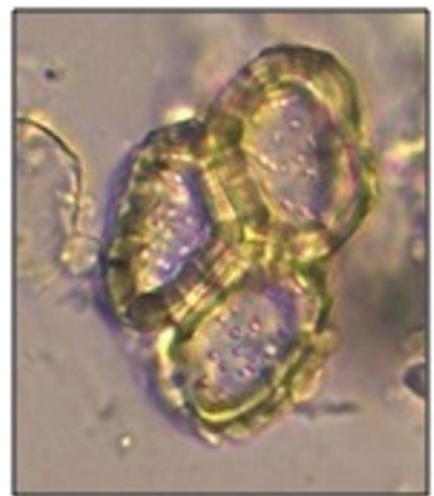

Figure 22: Represents group of stone cells respectively from AP III.

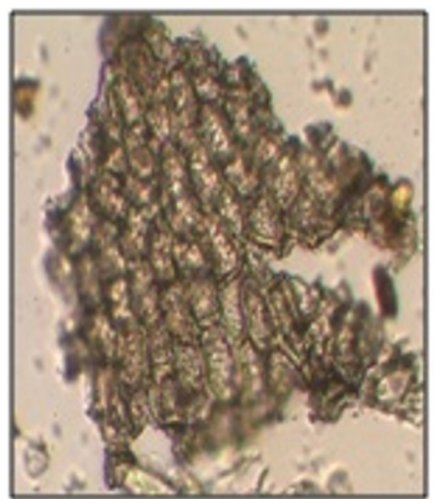

Figure 23: Represents group of stone cells from MP III.

Figure 24, 25 and 26 represents abundant rosette crystals, medullary rays and simple fiber fragment from NA IV. Figure 27 and 28 represents rosette and cluster rosette crystals from AP IV AND MP IV respectively.

However, all the three samples were completely devoid of stone cells and sclereids. Pongamia pinnata is a buff colored powder. Moderately fine powder was found to be rich in prismatic crystals whereas fine powder consisted of stone cells in large number Figure 29-32.

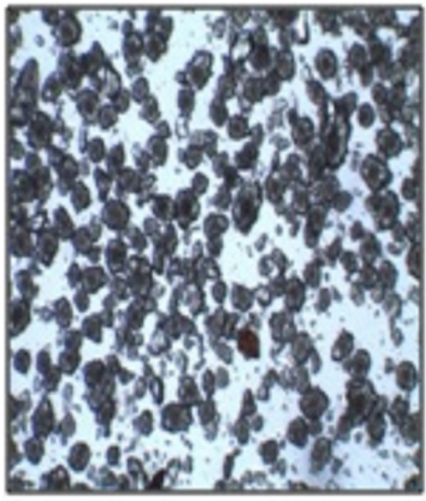

Figure 24: Represents abundant rosette crystals from NA IV.

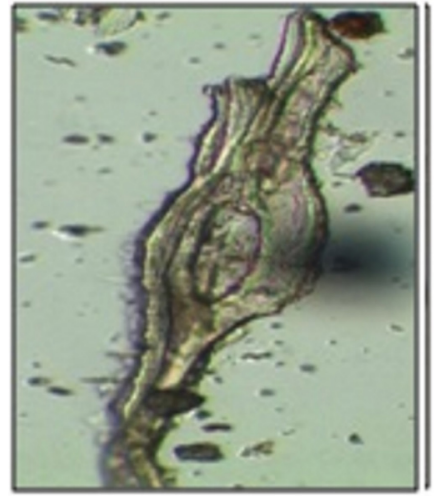

Figure 25: Represents medullary rays from NA IV.

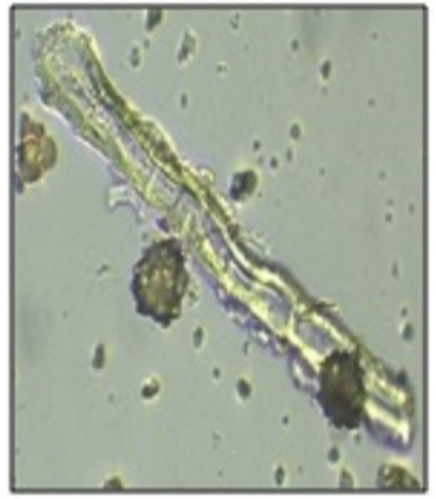

Figure 26: Represents simple fibre fragment from NA IV.

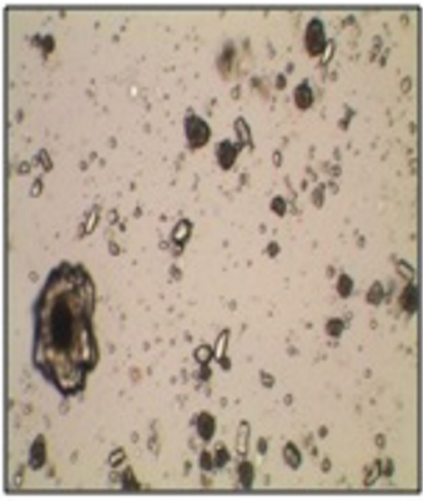

Figure 27: Represents rosette crystals from AP IV .

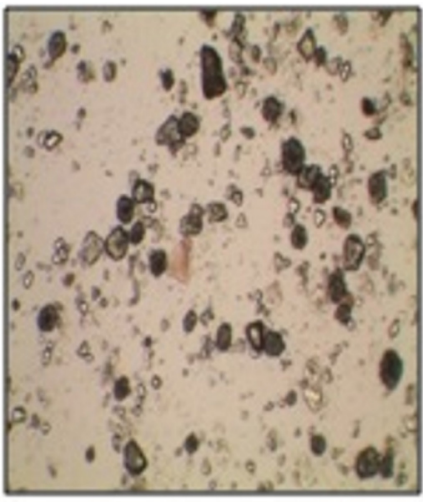

Figure 28: Represents cluster crystals from MP IV .

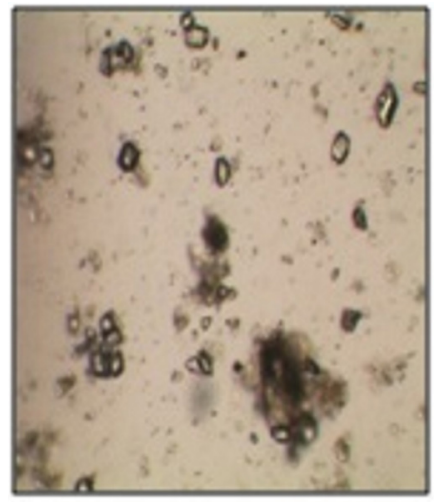

Figure 29: Represents prismatic crystals from NA V.

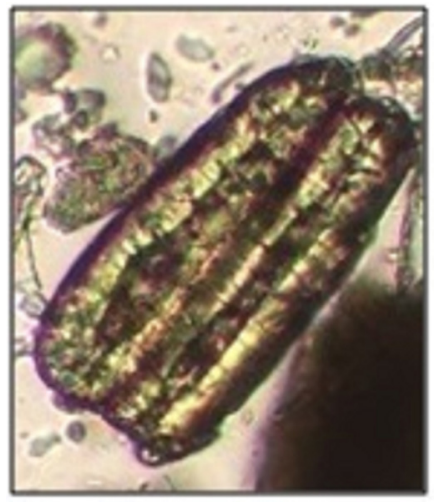

Figure 30: Represents stone cells from AP V.

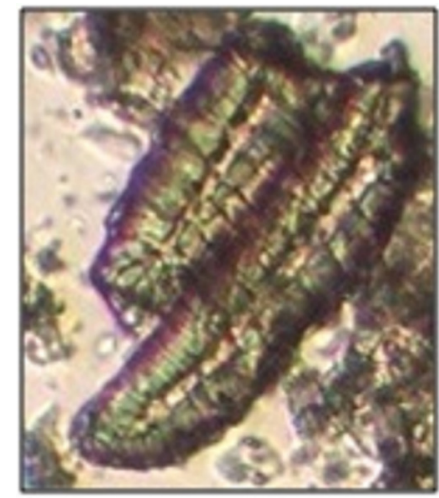

Figure 31: Represents stone cells from APV 


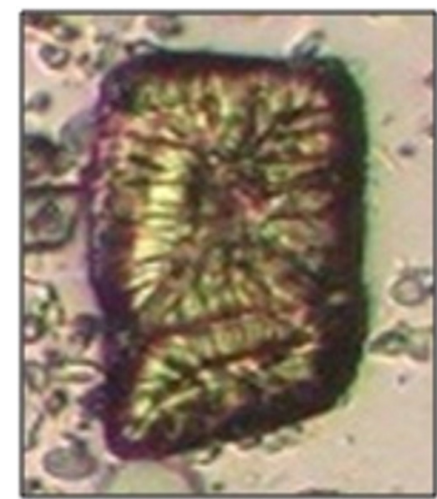

Figure 32: Represents stone cells from MPV .

Figure 29 represents prismatic crystals from NA V. Figure 30, 31 and 32 represents stone cells from AP V and MP V respectively.

\section{CONCLUSION}

The present study was aimed at development of standard operating protocol for preparation of slides to observe general as well as distinguishing microscopical characters of barks. From Table 2, grinding technique does not affect significantly probability of various microscopical features. Therefore, it can be concluded that powder microscopy can be used as one of the important parameter for quality control of any powdered material as it is quick, minimum expertise required and there is no need to cut and examine sections.

\section{ACKNOWLEDGEMENT}

The authors are grateful to Dr. Sunita Garg Scientist G, NISCAIR Pusa, New Delhi for her constant support in collection of plants with correct botanical identity.

\section{CONFLICT OF INTEREST}

We declare no conflict of interest.

\section{ABBREVIATION USED}

NISCAIR: National Institute of Science Communication and Information Resources; GMP: Good manufacturing Practices.

\section{REFERENCES}

1. Jhon-Jhen J. Application of Microscopic techniques for authentication of herbal drugs, Microscopy, science, technology, application and education, Formatex. 2010;803-12.

2. The Editorial Committee. Materia Medica of China. Shanghai: Shanghai Science and Technology Publishing House. 1999

3. European Pharmacopoeia Commission. European Pharmacopoeia. 6th Edition.

4. Strasbourg: European Directorate for the Quality of Medicines and Healthcare of Council of Europe. 2007.

5. British Pharmacopoeia Commission. British Pharmacopoeia 2009. London: The Stationery Office on Behalf of the Medicines and Healthcare Products Regulatory Agency. 2008

6. The United States Pharmacopeial Convention. The United States Pharmacopeia, 30 $0^{\text {th }}$ Revision / National Formulary. 25 $5^{\text {th }}$ Edition. Rochville: The United States Pharmacopeial Convention. 2005.

7. Japanese Pharmacopoeia Committee. The Japanese Pharmacopoeia. $15^{\text {th }}$ Edition. Tokyo: Society of Japanese Pharmacopoeia. 2006.

8. The Committee of the Ayurvedic Pharmacopoeia of India. The Ayurvedic Pharmacopoeia of India. New Delhi: The Controller of Publications Civil Lines; 2001.

9. Vietnamese Pharmacopoeia Commission, Ministry of Health. Vietnamese Pharmacopoeia. 2005;3rd.

10. Narayana DBA. Pharmacognosy and Technology. Pharmacognosy Magazine. 2010;6(23):145-6.

11. Dobriyal RM, Ananthanarayana DB. Controversial Nomenclature of Ayurvedic Drugs: Challenges for Scientists. Pharmacog Rev. 2009;3(5):1-7.

12. Chahuhan Malati G. Microscopic profile of powdered drugs used in Indian system of medicine, Bark Drugs, published by Institute of Ayurvedic Medicinal Plant Sciences, Jamnagar, Gujarat, India.1:124.

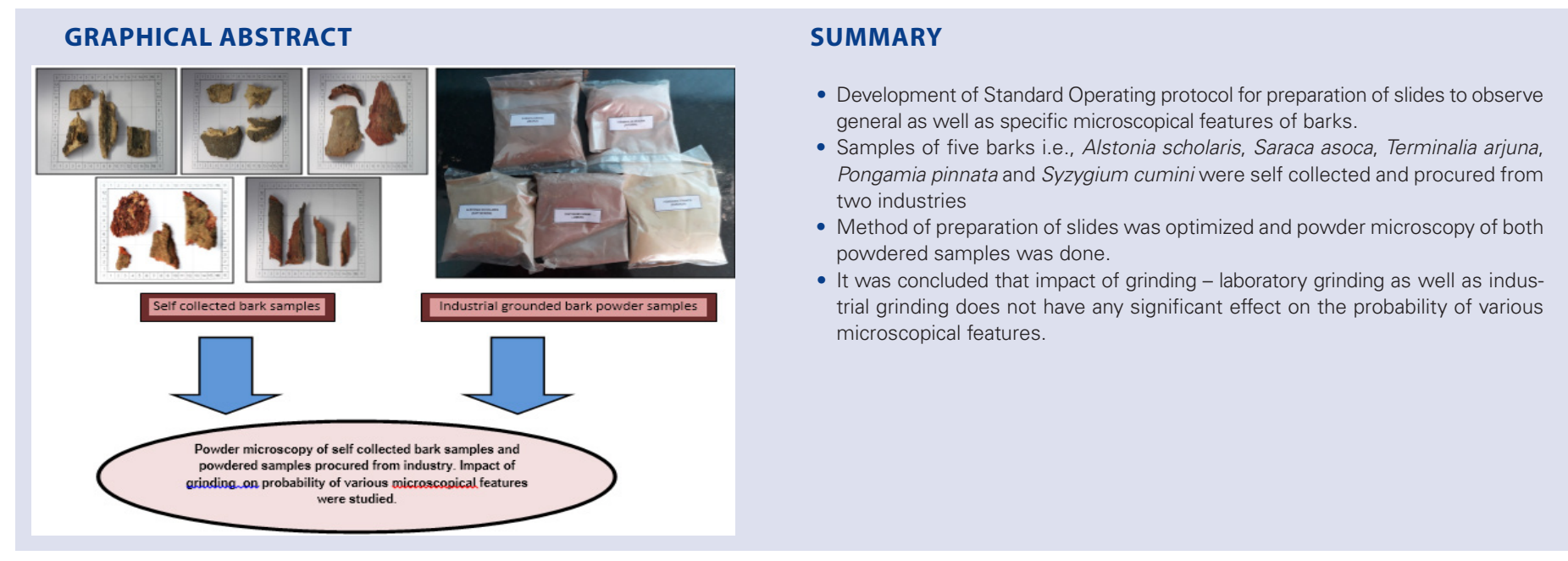

Cite this article: Singh D, Aeri V, Narayana DBA. Development of Standard Operating Protocol for Slide Preparation of Powdered Bark Samples with Varying Grinding Techniques. Pharmacog J. 2018;10(2):265-71. 